\title{
Impacto de la Personalidad y el Estilo Depresivo en los Resultados Psicoterapéuticos de Pacientes con Depresión
}

\section{The Impact of Personality and Depressive Style on the Psychotherapeutic Outcomes of Depressed Patients}

\author{
Paula Dagnino \\ Universidad Alberto Hurtado \\ Camila Valdés \\ Pontificia Universidad Católica de Chile \\ Isidora de la Fuente y María de los Ángeles Harismendy \\ Universidad Gabriela Mistral \\ Ana María Gallardo, Elyna Gómez-Barris y Guillermo de la Parra \\ Pontificia Universidad Católica de Chile
}

\begin{abstract}
La depresión es un trastorno heterogéneo que puede expresarse de manera diversa (estilo autocrítico o dependiente), presentando un funcionamiento estructural variado. El objetivo del estudio fue evaluar la relación entre el funcionamiento estructural y los estilos depresivos, junto con el efecto de estos sobre el cambio en los resultados durante la terapia. Se evaluaron intencionalmente 145 consultantes a 2 centros de atención psicoterapéutica ambulatoria de Santiago, Chile. Se midieron aspectos autocríticos y dependientes (DEQ), el funcionamiento estructural (OPD-SQ) al inicio de la terapia y los resultados (OQ-45.2) al inicio, $3^{\text {a }}$ y $6^{\text {a }}$ sesión de la terapia. Por medio de análisis correlacionales, de senderos y crecimiento latente, se encontró que mayor dependencia y autocrítica se relaciona con menor integración del funcionamiento estructural total. Se evidenció que a mayor dependencia menor integración en la percepción del self y manejo de la relación con los objetos y que a mayor autocrítica menor es la integración en vínculos internos. Alta dependencia o autocrítica se asocian con menor bienestar general inicial, aunque solo una alta dependencia se relaciona con un cambio positivo en los resultados.
\end{abstract}

Palabras clave: depresión, autocrítica, dependencia, estructura de personalidad, resultados psicoterapéuticos

\begin{abstract}
Depression is a heterogeneous disorder that can manifest itself in multiple ways (self-critical or dependent style) and whose structural functioning differs among patients. This study aimed to assess the relationship between structural functioning and depressive styles, along with the effect of the latter on changes in outcomes during therapy. Based on a purposive sample, 145 patients were evaluated at 2 outpatient psychotherapeutic care centers in Santiago, Chile. Several elements were measured: self-critical and dependent aspects (DEQ), structural functioning (OPD-SQ) at the start of the therapy, and outcomes (OQ-45.2) at the beginning of the process and during the $3^{\text {rd }}$ and $6^{\text {th }}$ sessions. Correlational, path, and latent growth analyses revealed that higher dependency and self-criticism levels are related to less integration of overall structural functioning. Higher dependency levels correlate with lower integration in the perception of self and in the management of object relations. Also, analyses showed that more self-criticism is linked to less integration of internal bonds. High dependency or self-criticism levels are associated with lower initial overall well-being; however, only high dependency correlates with a positive change in outcomes.
\end{abstract}

Keywords: depression, self-criticism, dependency, personality structure, psychotherapeutic outcome

Paula Dagnino, Facultad de Psicología, Universidad Alberto Hurtado, Facultad de Psicología, Universidad Gabriela Mistral y Departamento de Psiquiatría, Facultad de Medicina, Pontificia Universidad Católica de Chile; Camila Valdés y Ana María Gallardo, Escuela de Psicología, Pontificia Universidad Católica de Chile; Isidora de la Fuente y María de los Ángeles Harismendi, Facultad de Psicología, Universidad Gabriela Mistral; Elyna Gómez-Barris y Guillermo de la Parra, Departamento de Psiquiatría, Facultad de Medicina, Pontificia Universidad Católica de Chile.

Este estudio contó con el apoyo de la Comisión Nacional de Información Científica y Tecnológica, a través del Proyecto FONDECYT de Postdoctorado No 3140402 y del Proyecto FONDECYT Iniciación No 11170561, del Fondo de Innovación para la Competitividad del Ministerio de Economía, Fomento y Turismo de Chile, a través de la Iniciativa Científica Milenio, Fondo $\mathrm{N}^{\circ}$ IS130005, y del Proyecto N 10112 Capital Semilla de la Universidad Gabriela Mistral.

La correspondencia relativa a este artículo debe ser dirigida a Paula Dagnino, Facultad de Psicología, Universidad Alberto Hurtado, Almirante Barroso 26, Santiago, Chile. E-mail: pdagnino@uahurtado.cl 
La depresión es un síndrome de relevancia a nivel mundial debido a su alta prevalencia (Andrade et al., 2003). En Chile está por sobre un 17,2\% (Chile, Ministerio de Salud, 2013) y el suicidio, causado muchas veces por depresión, ha aumentado un 60\% entre 2000 y 2009 (Valdés \& Errázuriz, 2012). Además, es una de las principales causas de discapacidad en el mundo en términos de número total de años de vida. Resulta ser un síndrome relevante, ya que su impacto va más allá del paciente, teniendo importantes consecuencias para su círculo más cercano y la sociedad en su conjunto. De allí la relevancia de estudiar la depresión en mayor profundidad.

Un autor que resulta relevante para este estudio es Sydney Blatt, quien con su equipo (e.g., Blatt, 1974, 1991, 1995; Blatt \& Blass, 1990, 1996; Blatt \& Shichman, 1983) han propuesto dos polaridades de expresión de la depresión (mayormente dependiente o mayormente autocrítica). Este modelo surge, por un lado, a partir de las limitaciones de las clasificaciones descriptivas como el DSM-IV y, por otro, por la necesidad que clínicos e investigadores reconocen de contar con una perspectiva del desarrollo que permita comprender las alteraciones del funcionamiento psicológico actual. Esta aproximación evolutiva al desarrollo psicológico y psicopatológico entrega una visión longitudinal prospectiva para investigar la interacción biológica, psicológica y social que contribuye a la psicopatología que ocurre como variación o disrupción de un desarrollo normal. Es así que Blatt (2007, 2008) y sus colegas (Blatt \& Blass, 1990, 1996; Blatt \& Shichman, 1983) proponen que durante el desarrollo de los individuos se observan momentos de dependencia interpersonal y otros momentos de autodefinición, procesos que son fundamentales durante el desarrollo, y que su transcurso normal en el desarrollo se da de manera dialéctica. Sin embargo, disrupciones producto de eventos traumáticos o situaciones particulares (e.g., Fischer et al., 1997; Sroufe, 1997) llevarían al predominio de un estilo más dependiente o un estilo más autocrítico en la expresión de patologías posteriores como, por ejemplo, en la depresión.

En términos generales, este modelo plantea que durante el desarrollo se dan estos dos estilos en un diálogo constante y que poseen características particulares; es así como un estilo dependiente se caracteriza porque su "tarea vital" es estar en relación con otros; en cambio, en el autocrítico sería la de auto definirse, de buscar mantener permanentemente una definición del sí mismo. El estilo dependiente se define en base a la relación con los otros, a cómo se están relacionando. En el caso de los autocríticos, estos se definen en base a la autonomía, al control de sí mismo y los demás. La autoestima es regulada en los dependientes a través de si son queridos o no; en cambio, en los autocríticos la autoestima se regula según si reciben reconocimiento, admiración y respeto (Blatt, Shahar \& Zuroff, 2002). Los dependientes muestran un intenso anhelo por ser amados, nutridos y protegidos y evalúan a los otros según la capacidad inmediata de cuidar y proveer satisfacción; en cambio, para los autocríticos no es una prioridad establecer ni mantener relaciones interpersonales; suelen ser personas más bien solitarias, reservadas o distantes. En cuanto al discurso, el dependiente refiere sentimientos, afectos y relaciones con otros; en cambio, el autocrítico habla de situaciones concretas, de conducta manifiesta, presentando una constante autoevaluación y severa autocrítica. Ambos estilos se han evidenciado en estudios como el de Luyten y Blatt (2013).

En términos de la psicopatología, específicamente en la depresión, cada estilo muestra diferencias. Es así que lo que provoca que los dependientes se depriman son las separaciones, el duelo y el abandono o rechazo y esto lleva a que durante la psicoterapia muestren sentimientos de tristeza, soledad y desesperanza (Blatt, D'Afflitti \& Quinlan, 1976; Luyten \& Blatt, 2013). En el caso del autocrítico, lo que provoca la depresión son situaciones de humillación, fracaso e injuria a la autoestima y se manifiesta a través de autorreproches, culpa y sentimientos de fracaso y de inferioridad (Blatt, 2008). Estudios que han utilizado esta diferenciación han mostrado que tanto los pacientes autocríticos como los dependientes (Blatt, 1992; Blatt et al., 1994; Blatt, Ford, Berman, Cook \& Meyer, 1988) muestran diferentes necesidades, responden de manera distinta a las intervenciones terapéuticas y evidencian diferentes resultados. Por ello es que el funcionamiento de cada estilo depresivo (autocrítico o dependiente) es distinto. El concepto general de funcionamiento se relaciona usualmente con el concepto de estructura u organización de la personalidad. Mientras la estructura u organización representa los conceptos teóricos (la disponibilidad de las capacidades), el concepto de funcionamiento de personalidad se refiere a las manifestaciones observables de las condiciones estructurales (el uso actual de las capacidades) (Dahlbender, Rudolf \& OPD Task Force, 2006). Considerar el funcionamiento o la utilización o no de ciertas capacidades de manera específica para cada estilo depresivo puede favorecer una comprensión más acabada de estas polaridades, así como desarrollar intervenciones dirigidas y, por ende, centradas en el paciente.

Una manera de aproximarse al funcionamiento de los pacientes es la que plantea el Diagnóstico Psicodinámico Operacionalizado (OPD-2; Grupo de Trabajo OPD, 2008). Este diagnóstico surge en los años 
90 de un grupo de psicoterapeutas alemanes, con el objetivo de ampliar la clasificación de diagnósticos mentales basados en descripción de síntomas, agregando algunas dimensiones dinámicas fundamentales. Se organiza de manera multiaxial, permitiendo identificar distintas áreas de evaluación, en las cuales pueden focalizarse las intervenciones psicoterapéuticas.

De hecho, una de las dimensiones que evalúa el OPD-2 es el funcionamiento estructural. Esta dimensión incorpora aproximaciones de las relaciones de objeto, del self y de la psicología del yo (Rudolf, Oberbracht \& Grande, 1998). Para el OPD-2, la estructura es considerada como un tejido de disposiciones psíquicas que determina un estilo personal y relativamente permanente a través del cual el individuo puede recuperar (o no) su equilibrio intrapsíquico e interpersonal frente a las demandas del medio interno y externo. Debido a la relevancia que tiene este eje para la comprensión del paciente, es que ha decantado en un instrumento de autorreporte (OPD-SQ; Ehrenthal et al., 2012), el cual describe cualidades o deficiencias en el funcionamiento estructural, tanto en su orientación hacia el sí mismo (self) como hacia los otros (objetos), descomponiéndose en ocho funciones (ver Tabla 1): (a) percepción del self, (b) percepción de los objetos, (c) manejo del self, (d) manejo de la relación con los objetos, (e) comunicación emocional hacia adentro, (f) comunicación emocional hacia afuera, $(\mathrm{g})$ vínculo interno y $(\mathrm{h})$ vínculo con relaciones externas. Cada función puede definirse en cuatro niveles de integración (bien integrada, moderadamente integrada, escasamente integrada, desintegrada). Las distintas funciones estructurales pueden constituir un foco terapéutico (Ehrenthal et al., 2012). Debido a que las funciones estructurales están definidas de manera cercana a la conducta observable, la dimensión Estructura demuestra tener una buena aplicabilidad clínica (Grupo de Trabajo OPD, 2008) y la focalización en este eje ha mostrado su utilidad en variados ámbitos, llevando al desarrollo de un manual de psicoterapia con recomendaciones específicas acerca del trabajo terapéutico (Rudolf, 2013).

Tabla 1

Funciones Estructurales en OPD Dirigidas hacia el Self y hacia los Otros

\begin{abstract}
1. Percepción del self
Capacidad de percibir de manera diferenciada una imagen del propio self y de los sucesos intrapsíquicos, especialmente de los afectos.

\section{Manejo del self}

Aspecto integrador de la vivencia psíquica. De ello resulta la capacidad de responsabilizarse como el gestor de las propias acciones competentes; de esta autoeficacia deriva la confianza en sí mismo y la autoafirmación.

5. Comunicación emocional hacia adentro

Capacidad de llevar diálogos internos y de entenderse uno mismo. La capacidad de dejar que surjan los afectos en uno y de vivirlos es un requisito para lograr esa comunicación.
\end{abstract}

\section{Vínculo interno}

Capacidad de relacionarse con otros, tanto intrapsíquicamente como en el contacto interpersonal. El punto de partida lo genera la capacidad de crear representaciones internas de personas significativas. Se refiere a la capacidad de internalización.

\section{Percepción de los objetos}

Capacidad de desarrollar una imagen realista del interlocutor. En especial, consiste en poder percibir al otro como poseedor de características individuales.

4. Manejo de la relación con los objetos

Las tareas de regulación frente al objeto incluyen tanto la protección de la relación frente a los propios impulsos como el resguardo de los propios intereses, para que estos no se pierdan ante la influencia de otros.

6. Comunicación emocional hacia afuera

Se refiere al intercambio emocional entre el self y el otro. Alude al establecimiento de contacto emocional entre personas, la comunicación de los propios afectos y la capacidad de dejarse tocar emocionalmente por los afectos de otros, así como la comprensión mutua y el sentimiento del "nosotros" de la reciprocidad.

8. Vínculo con relaciones externas

Capacidad para poder vincularse emocionalmente a otros en relaciones reales. Afectos como la solidaridad, el sentido de responsabilidad, la culpa, entre otros, son afectos vinculares.

Estudios realizados a partir de este eje han encontrado una correlación inversa entre la integración del funcionamiento estructural y el tiempo de enfermedad psicógena, lo que podría explicarse por una capacidad deficitaria en la función de manejo del self y de los otros (Rudolf, Grande, Oberbracht \& Jakobsen, 1996). Por otro lado, considerando la relación de funcionamiento estructural y diagnósticos específicos, los estudios se han centrado en los trastornos de la personalidad. Doering et al. (2014) encontraron que estos pacientes mostraban un funcionamiento significativamente peor que aquellos que no presentaban trastorno de la personalidad. Específicamente, los pacientes con trastornos de la personalidad del cluster B eran los que se 
observaban con mayores dificultades en su funcionamiento estructural que los del cluster C. El funcionamiento estructural medido a través del OPD muestra asociación con medidas de personalidad y de apego. Se observan diferencias entre una población normal y una clínica, así como también entre diferentes muestras clínicas con variadas dificultades en la personalidad (e.g., pacientes con o sin trastorno de la personalidad; Ehrenthal et al., 2012). Por último, en un estudio con pacientes depresivos, Dagnino, GómezBarris, Gallardo, Valdés y de la Parra (2017) encontraron que a mayor sintomatología depresiva peor es el funcionamiento estructural.

Establecer el funcionamiento estructural de los pacientes con estilos depresivos autocrítico o dependiente resulta útil para describir con mayor profundidad las particularidades de cada uno, pero, aún más, para identificar aquellas funciones que presentan mayor vulnerabilidad en cada estilo.

Considerar las particularidades de los estilos depresivos en cuanto a su funcionamiento estructural es clínicamente relevante. Por otro lado, varios estudios han mostrado que los estilos depresivos no solo difieren en ciertos aspectos, sino también cómo evolucionan durante el proceso. Blatt (1992), reanalizando los datos del Proyecto Menninger de Investigación en Psicoterapia (Wallerstein, 1986), encontró que los pacientes dependientes presentaban mayor cambio positivo cuando se exponían a psicoterapia y no a psicoanálisis; en cambio, los pacientes autocríticos presentaban mayor cambio positivo en psicoanálisis que en psicoterapia. Otros estudios (Blatt et al., 1998; Blatt, Quinlan, Pilkonis \& Shea, 1995; Blatt, Zuroff, Quinlan \& Pilkonis, 1996; Zuroff et al., 2000) mostraron que los pacientes con alta autocrítica inicial predecían peores resultados en la terminación (esto es, después de 16 semanas de tratamiento), así como también a los 18 meses después de haber terminado el proceso (Blatt et al., 1995; Blatt et al., 1998). Análisis posteriores mostraron que el nivel de autocrítica inicial impedía el progreso terapéutico en dos tercios de la muestra, específicamente en la segunda mitad del tratamiento, entre la $9^{\mathrm{a}}$ y $12^{\mathrm{a}}$ sesión (Blatt et al., 1998).

A pesar de la evidencia, los estudios mencionados presentan ciertas limitaciones, ya que algunos de ellos evalúan los resultados de la psicoterapia al término de esta (e.g., Blatt et al., 1995) o en poblaciones de sujetos con diagnósticos graves, como esquizofrenia, u hospitalizados en centros especiales (e.g., Blatt et al., 1998). De allí que resulta relevante evaluar el efecto de los estilos depresivos (autocrítico o dependiente) en los resultados psicoterapéuticos durante la psicoterapia. Se espera que la evolución de los pacientes en cuanto a sus resultados muestre trayectorias distintas. De hecho, se espera que los pacientes con un estilo autocrítico tengan una trayectoria de cambio menor que los dependientes.

En términos generales, este estudio buscó evaluar la relación entre el funcionamiento estructural y los estilos depresivos, junto con el efecto de estos sobre el cambio en los resultados durante la terapia. El primer objetivo fue establecer de qué manera las distintas funciones estructurales determinan los diferentes estilos de depresión (dependiente y autocrítica), identificando qué vulnerabilidades se encuentran más presente en cada una de ellas. Un segundo objetivo fue evaluar el efecto de los estilos depresivos (autocrítico o dependiente) sobre el cambio en los resultados psicoterapéuticos.

En cuanto al primer objetivo, se esperaba que los pacientes que presentan estilos depresivos (dependiente o autocrítico) presenten dificultades en el funcionamiento estructural, pero, además, que se pudieran identificar vulnerabilidades en algunas funciones específicas para cada una de las formas de expresar la depresión. Particularmente, se esperaba que pacientes que experimentan la depresión en forma autocrítica presenten vulnerabilidades en las funciones relacionadas con el self, mientras que los pacientes que experimentan una depresión con un estilo dependiente presenten vulnerabilidad principalmente en las funciones relacionadas con los otros. Para el segundo objetivo, se esperaba que los estilos depresivos muestren evoluciones distintas de los resultados terapéuticos, específicamente, se esperaba que la presencia de autocrítica tenga un efecto negativo en el cambio terapéutico durante el proceso, no así la dependencia.

Esta línea de investigación que toma en cuenta las características específicas de cada paciente y su respuesta al tratamiento en psicoterapia resulta relevante, ya que los pacientes con depresión son heterogéneos y, por lo tanto, se debe establecer una ayuda psicoterapéutica diferencial y adecuada a cada uno de ellos (Luyten \& Blatt, 2011). Para ello se debe contar con una descripción más precisa y completa del paciente, tomando en cuenta sobre todo aquellas disfunciones que deben considerarse como focos del tratamiento, como son las vulnerabilidades estructurales (Corveleyn, Luyten \& Blatt, 2005; van Praag, 2010). Solo así se podrán desarrollar estrategias centradas en el paciente y no en el síndrome (de la Parra, Dagnino, Valdés \& Krause, 2017). 


\section{Método}

\section{Participantes}

Se contó con una muestra no probabilística intencional de 145 pacientes consultantes a psicoterapia del Centro de Atención Psicológica de la Universidad Gabriela Mistral (CAP UGM) y de la Unidad de Psicoterapia Adultos de la Unidad de Salud Mental, San Joaquín, de la Pontificia Universidad Católica de Chile (UPA UC). El criterio de inclusión fue la presencia de sintomatología depresiva (medida a través del cuestionario BDI) y los criterios de exclusión fueron pacientes menores de 18 años, consultantes por abuso de sustancia, con síntomas psicóticos, disfunción cognitiva o trastorno alimentario.

El mecanismo de invitación de los participantes dependió del centro donde asistió. En el CAP UGM fue la secretaria quien contactó a los pacientes y realizó la invitación a participar en la investigación, mientras que en la UPA UC fueron dos asistentes de investigación quienes concurrieron al centro para invitar de forma presencial a los participantes.

En general, el promedio de edad fue de 31,11 años $(D E=11,22)$. El $74 \%(n=106)$ de los participantes eran mujeres. Cabe mencionar que las psicoterapias realizadas en ambos centros son en modalidad individual, de distinta orientación teórica (psicodinámica, sistémica y cognitivo conductual) y de tiempo limitado (promedio $=15$ sesiones, $D E=2)($ ver Tabla 2$)$.

Tabla 2

Estadísticos Descriptivos de la Muestra Total y de las Dos Submuestras que la Conforman

\begin{tabular}{|c|c|c|c|c|}
\hline & Variable & CAP UGM & UPA UC & Total \\
\hline$n$ & & 71 & 74 & 145 \\
\hline Mujeres: \% & & 71,8 & 76,4 & 74,1 \\
\hline Edad: promedio $(D E)$ & & $28,86(10,82)$ & $34,04(11,16)$ & $31,11(11,22)$ \\
\hline \multicolumn{5}{|l|}{ Escolaridad: \% } \\
\hline & Media incompleta & 0,0 & 4,1 & 2,0 \\
\hline & Media completa & 33,3 & 26,5 & 30,0 \\
\hline & Técnico-profesional & 15,7 & 10,2 & 13,0 \\
\hline & Universitaria & 51,0 & 59,2 & 55,0 \\
\hline \multicolumn{5}{|c|}{ OQ inicial: promedio $(D E)$} \\
\hline & Total & $69,09(27,11)$ & $82,60(26,95)$ & $75,99(27,77)$ \\
\hline & Escala de Rol Social & $12,47(4,87)$ & $14,44(5,88)$ & $13,48(5,48)$ \\
\hline & Escala de Relaciones Interpersonales & $15,21(7,35)$ & $17,97(6,32)$ & $16,62(6,96)$ \\
\hline & Escala de Síntomas & $41,40(17,77)$ & $50,19(17,38)$ & $45,89(18,06)$ \\
\hline \multicolumn{5}{|c|}{ DEQ inicial: promedio $(D E)$} \\
\hline & Autocrítica & $0,02(1,20)$ & $0,46(1,00)$ & $0,26(1,11)$ \\
\hline & Dependencia & $-0,46(0,95)$ & $-0,25(1,22)$ & $-0,35(1,10)$ \\
\hline \multicolumn{5}{|c|}{ OPD-SQ Inicial: promedio $(D E)$} \\
\hline & Percepción del Self & $1,88(1,14)$ & $2,23(1,18)$ & $2,06(1,17)$ \\
\hline & Percepción de los Objetos & $1,65(0,90)$ & $1,90(0,82)$ & $1,78(0,87)$ \\
\hline & Manejo del Self & $1,84(0,96)$ & $2,16(0,93)$ & $2,00(0,96)$ \\
\hline & Manejo de la Relación con los Objetos & $1,68(0,86)$ & $1,95(0,81)$ & $1,82(0,84)$ \\
\hline & Comunicación Emocional hacia Adentro & $1,55(0,68)$ & $1,90(0,84)$ & $1,73(0,78)$ \\
\hline & Comunicación Emocional hacia Afuera & $1,76(0,79)$ & $2,06(0,82)$ & $1,91(0,82)$ \\
\hline & Vínculo Interno & $1,65(1,17)$ & $2,19(1,03)$ & $1,92(1,13)$ \\
\hline & Vínculo Relaciones Externas & $2,34(0,73)$ & $2,50(0,85)$ & $2,42(0,79)$ \\
\hline
\end{tabular}

\section{Instrumentos}

Inventario de Depresión de Beck (BDI-IA; Beck, Ward, Mendelson, Mock \& Erbaugh, 1961). Es un cuestionario de autorreporte que permite evaluar sintomatología depresiva. El BDI-IA ha sido traducido a diversos idiomas y es utilizado ampliamente en todo el mundo y en atención primaria en Chile (Alvarado, Vega, Sanhueza \& Muñoz, 2005). El instrumento consta de 21 ítems puntuados en una escala de 0 a 3, donde 
a mayor puntaje mayor presencia de sintomatología depresiva. Un ejemplo de item es: (0) No me siento triste, (1) Me siento triste, (2) Me siento triste continuamente y no puedo dejar de estarlo, (3) Ya no puedo soportar esta pena. Los puntajes de corte estándar son los siguientes: 0-9 depresión mínima, 10-18 depresión leve, 19-29 depresión moderada y 30-63 depresión severa. Presenta adecuada consistencia interna, con un coeficiente alfa de Cronbach de 0,86 para población clínica y 0,81 para población no clínica (Beck, Steer \& Carbin, 1988). La validación realizada en Chile muestra que es un instrumento apropiado para evaluar sintomatología depresiva, con un alto grado de consistencia interna $(\alpha=0,93)$ y que puede distinguir entre pacientes depresivos y no-depresivos (Valdés et al., 2017).

Cuestionario de Experiencias Depresivas (DEQ; Blatt et al., 1976; Blatt et al., 1995; Zuroff, Quinlan \& Blatt, 1990). Este cuestionario fue usado para evaluar grado de dependencia y autocrítica. Incluye ítems que son representativos de experiencias comunes de individuos que presentan depresión, sin focalizarse en los síntomas depresivos. El DEQ contiene 66 ítems, 25 de ellos apuntan a autocrítica (e.g., Fijo mis metas y objetivos lo más alto posible) y otros 25, a dependencia (e.g., Sin el apoyo de los que están cerca de mí, me sentiría desamparado/a); el resto de los ítems aluden a un factor llamado Eficacia, que, para efectos de este estudio, no fue considerado por no mostrar un comportamiento estable. Todos los ítems están estructurados en una escala Likert entre 1 (en total desacuerdo) hasta 7 (en total acuerdo). El DEQ entrega puntuaciones factoriales para los tres factores principales, Dependencia, Autocrítica y Eficacia, y cada uno de los ítems contribuye a los tres factores. Debido a que los puntajes que se derivan para cada uno de los tres factores son puntuaciones factoriales, la media de cada factor es cero y la desviación estándar, +/- 1. Las puntuaciones mínimas indican baja participación en ese factor en particular. Las propiedades psicométricas del DEQ son satisfactorias, con altas estimaciones de consistencia interna ( $\alpha=0,81$ para Dependencia, $\alpha=0,75$ para Autocrítica y $\alpha=0,73$ para Eficacia) y confiabilidad test-retest (Blatt et al., 1976; Zuroff et al., 1990). Aunque este instrumento se encuentra en proceso de validación en Chile (Pérez et al., 2018), varios son los estudios a nivel nacional que han utilizado con éxito el instrumento, al relacionarlo, por ejemplo, con funcionamiento estructural (Dagnino, Gómez-Barris et al., 2017; de la Parra et al., 2017) y con estilo de apego, sintomatología depresiva y apoyo social (Dagnino, Pérez, Gómez, Gloger \& Krause, 2017), mostrando que este se relaciona con las variables en la dirección esperada.

Cuestionario para Evaluación de Resultados y Evolución en Psicoterapia (OQ-45.2). Este cuestionario (Lambert et al., 1996) es un autorreporte que mide los resultados psicoterapéuticos a través de tres áreas: (a) sintomatología (e.g., Me canso rápidamente), (b) relaciones interpersonales (e.g., Me llevo bien con otros) y (c) rol social (e.g., Me siento presionado [estresado] en el trabajo/escuela). Consiste en 45 ítems; cada ítem se puntúa de 1 (nunca) a 4 (casi siempre) puntos en una escala Likert, de manera que el rango del puntaje puede ir de 0 a 180 puntos. A mayor puntaje mayor disconfort psicológico. Fue validado en Chile (de la Parra \& von Bergen, 2001, Junio; de la Parra, von Bergen \& del Río, 2002), encontrándose un punto de corte de 73 (que diferencia a la población normal de la clínica) y un índice de cambio confiable de 17 . La confiabilidad test-retest fue de 0,90 (para el $\mathrm{OQ}$ total) y el alfa de Cronbach, de 0,91 tanto para la muestra clínica como no clínica.

\section{Cuestionario de Funcionamiento Estructural del Diagnóstico Psicodinámico} Operacionalizado (OPD-SQ; Ehrenthal et al., 2012). Es un cuestionario de autorreporte que evalúa el funcionamiento estructural de acuerdo al sistema OPD (Grupo de Trabajo OPD, 2008). Consiste en 95 ítems, puntuados en una escala de 1 a 5 puntos $(1=$ Totalmente en desacuerdo; $5=$ Totalmente de acuerdo), que pretenden cubrir las ocho funciones psíquicas descritas en la Tabla 1: percepción del self (12 ítems), percepción de los objetos (17 ítems), manejo del self (13 ítems), manejo de la relación con los objetos (12 ítems), comunicación emocional hacia adentro (11 ítems), comunicación emocional hacia afuera (14 ítems), vínculo interno (8 ítems) y vínculo relaciones externas (8 ítems). En la Tabla 3 se incluyen las subfunciones de cada subescala, el número de ítems para cada una y algunos ejemplos. El promedio de las subescalas da cuenta del puntaje de la estructura total. Mayores puntajes indican mayores dificultades en las funciones estructurales. Un estudio de consistencia interna en 1110 sujetos mostró un alfa de Cronbach de 0,71 a 0,91 para las subescalas y un alfa de Cronbach de 0,96 para la escala total en tres muestras: $N=734$ no clínicos, $N=172$ pacientes ambulatorios y $N=204$ residentes en unidades de salud mental (Ehrenthal et al., 2012). El OPD-SQ se correlaciona en la dirección esperada con otras medidas de personalidad, como el apego, la cantidad de diagnósticos eje II del DSM-IV y con evaluaciones de expertos (Dinger et al., 2014). Actualmente 
se encuentra adaptado en Chile y ha comenzado su validación por de la Parra et al. (2018), artículo publicado en este mismo número.

Tabla 3

Funciones Estructurales en OPD-SQ Dirigidas hacia el Self y hacia los Otros

\begin{tabular}{|c|c|c|c|}
\hline Subescala & Subfunción incluida & $\mathrm{N}^{\circ}$ de ítems & Ítem de ejemplo \\
\hline \multirow[t]{3}{*}{ Percepción del Self } & Autorreflexión & 4 & Me resulta muy difícil describirme a mí mismo/a. \\
\hline & $\begin{array}{l}\text { Diferenciación } \\
\text { afectiva }\end{array}$ & 4 & $\begin{array}{l}\text { A menudo no tengo claro qué es exactamente lo que } \\
\text { estoy sintiendo. }\end{array}$ \\
\hline & Identidad & 4 & A veces afloran cosas en mí que no calzan conmigo. \\
\hline \multirow[t]{3}{*}{ Percepción de los Objetos } & $\begin{array}{l}\text { Diferenciación self- } \\
\text { objeto }\end{array}$ & 7 & $\begin{array}{l}\text { A veces dudo si alguien está pensando algo de mí o si } \\
\text { solo es mi imaginación. }\end{array}$ \\
\hline & $\begin{array}{l}\text { Percepción de objeto } \\
\text { total }\end{array}$ & 4 & $\begin{array}{l}\text { O la otra persona está en mi misma onda o no vamos a } \\
\text { funcionar. }\end{array}$ \\
\hline & $\begin{array}{l}\text { Percepción realista } \\
\text { del objeto }\end{array}$ & 6 & $\begin{array}{l}\text { Los demás me dicen que siempre vuelvo a elegir a los } \\
\text { amigos equivocados. }\end{array}$ \\
\hline \multirow[t]{3}{*}{ Manejo del Self } & Manejo de impulsos & 4 & $\begin{array}{l}\text { A veces estoy tan furioso que no puedo responder por } \\
\text { lo que hago. }\end{array}$ \\
\hline & Tolerancia afectiva & 5 & $\begin{array}{l}\text { Mis emociones son a veces tan intensas que me } \\
\text { asustan. }\end{array}$ \\
\hline & $\begin{array}{l}\text { Regulación de la } \\
\text { autoestima }\end{array}$ & 4 & $\begin{array}{l}\text { Cuando alguien me critica, me resulta difícil } \\
\text { superarlo. }\end{array}$ \\
\hline \multirow[t]{2}{*}{$\begin{array}{l}\text { Manejo de la Relación con los } \\
\text { Objetos }\end{array}$} & $\begin{array}{l}\text { Regulación de la } \\
\text { relación con el objeto }\end{array}$ & 6 & $\begin{array}{l}\text { Cuando me enojo, tiendo hacer daño en mis } \\
\text { relaciones. }\end{array}$ \\
\hline & Anticipación & 6 & $\begin{array}{l}\text { A veces juzgo mal cómo mi conducta afecta a los } \\
\text { demás. }\end{array}$ \\
\hline \multirow{2}{*}{$\begin{array}{l}\text { Comunicación Emocional hacia } \\
\text { Adentro }\end{array}$} & Vivencia de los afectos & 4 & Me cuesta percibir mis emociones. \\
\hline & $\begin{array}{l}\text { Uso de las fantasías } \\
\text { Self corporal }\end{array}$ & $\begin{array}{l}3 \\
4\end{array}$ & $\begin{array}{l}\text { Mis fantasías e ideas me vitalizan y enriquecen. } \\
\text { A menudo soy incapaz de percibir bien mi cuerpo. }\end{array}$ \\
\hline \multirow[t]{3}{*}{$\begin{array}{l}\text { Comunicación Emocional hacia } \\
\text { Afuera }\end{array}$} & Establecer contacto & 4 & $\begin{array}{l}\text { Me resulta difícil establecer contacto con otras } \\
\text { personas. }\end{array}$ \\
\hline & $\begin{array}{l}\text { Comunicación de los } \\
\text { afectos }\end{array}$ & 6 & $\begin{array}{l}\text { Me han dicho que muestro muy poco mis } \\
\text { sentimientos. }\end{array}$ \\
\hline & Empatía & 4 & $\begin{array}{l}\text { Cuando alguien lo está pasando mal, suelo } \\
\text { preocuparme. }\end{array}$ \\
\hline \multirow[t]{2}{*}{ Vínculo Interno } & Internalización & 4 & $\begin{array}{l}\text { A menudo tiendo a pensar en ciertas personas que } \\
\text { podrían dañarme. }\end{array}$ \\
\hline & Uso de introyectos & 4 & Me resulta difícil hacer algo bueno para mí. \\
\hline \multirow[t]{2}{*}{ Vínculo Relaciones Externas } & Aceptar ayuda & 4 & Me resulta difícil pedir ayuda a los demás. \\
\hline & $\begin{array}{l}\text { Desprenderse de } \\
\text { vínculos, separarse }\end{array}$ & 4 & $\begin{array}{l}\text { Las separaciones y despedidas son muy difíciles para } \\
\text { mí. }\end{array}$ \\
\hline
\end{tabular}

Ficha sociodemográfica. Consiste en una serie de preguntas acerca de datos del paciente que él mismo debe contestar. Se incluyen las siguientes infomaciones: edad, sexo, estado civil, nivel de estudios alcanzados y ocupación.

\section{Procedimiento}

Este estudio fue aprobado por el Comité Ético Científico de Ciencias Sociales, Artes y Humanidades de la Pontificia Universidad Católica de Chile (ID 160104007).

En ambas instituciones los pacientes - contactados en terreno por ayudantes de investigación-firmaron una carta de consentimiento de participación en el estudio, en la que se les explicó la relevancia de la investigación y los datos que serían requeridos, señalando que se salvaguardarían todos los antecedentes 
personales. Asimismo, se les explicó que su participación era voluntaria y que se podían retirar de la investigación cuando ellos estimaran necesario, sin poner en riesgo su proceso terapéutico.

No hubo ninguna entrega de retribución económica a los participantes.

A los que accedieron a participar (un 98\% de los invitados) se les entregó el BDI (sintomatología depresiva) para contestar en ese momento. Al ser un instrumento corto, fue codificado inmediatamente por el ayudante de investigación presente, para asegurar que el paciente cumpliera con el requisito para ingresar al estudio. De no cumplirlo, se le explicaba y agradecía su buena disposición, pero no ingresaba al estudio. Cuando el paciente cumplía con el criterio de inclusión (un 88\% de los pacientes que aceptaron participar), se le aplicó el cuestionario OQ-45.2 (bienestar general) y se le entregó en un sobre una ficha sociodemográfica y los instrumentos OPD-SQ (funcionamiento estructural) y DEQ (dependencia o autocrítica) para que los contestara en su casa y lo devolviera en la segunda sesión. El cuestionario OQ-45.2 fue administrado nuevamente - por el ayudante de investigación de manera presencial- previo a la tercera y sexta sesión.

\section{Análisis de Datos}

En primera instancia se calcularon correlaciones bivariadas de Pearson para evaluar el nivel de asociación del puntaje total del OPD-SQ y de sus subescalas con el nivel de dependencia, autocrítica (ambos medidos mediante el DEQ) y el bienestar general de los participantes en la primera medición. Estos análisis fueron realizados con el software estadístico R 3.1.2 (R Core Team, 2008).

Luego, se realizó un análisis de senderos, en el que las distintas funciones del OPD-SQ fueron utilizadas como predictores de los puntajes en las escalas de dependencia y autocrítica del DEQ (ver Figura 1). La correlación entre autocrítica y dependencia fue fijada en 0 , dada la naturaleza ortogonal que presentan ambas variables (Blatt, 2008). En esta instancia, se utilizaron datos transversales obtenidos en la entrega de cuestionarios previo al inicio de la terapia. Se utilizó como método de estimación el método maximum likelihood robusto, para compensar la falta de normalidad multivariada. Como medidas de bondad de ajuste del modelo se incluyeron medidas de ajuste absoluto del modelo, como la raíz del error cuadrático medio de aproximación (RMSEA) y la raíz del residuo cuadrático medio estandarizado (SRMR), y una medida de ajuste incremental, el índice comparativo de Bentler-Bonett (CFI), como es propuesto por Jackson, Gillaspy Jr. y Purc-Stephenson (2009), considerando que el modelo presenta un buen ajuste si se presenta un RMSEA $<0,05$, un CFI $>0,95$ y un SRMR $<0,05$.

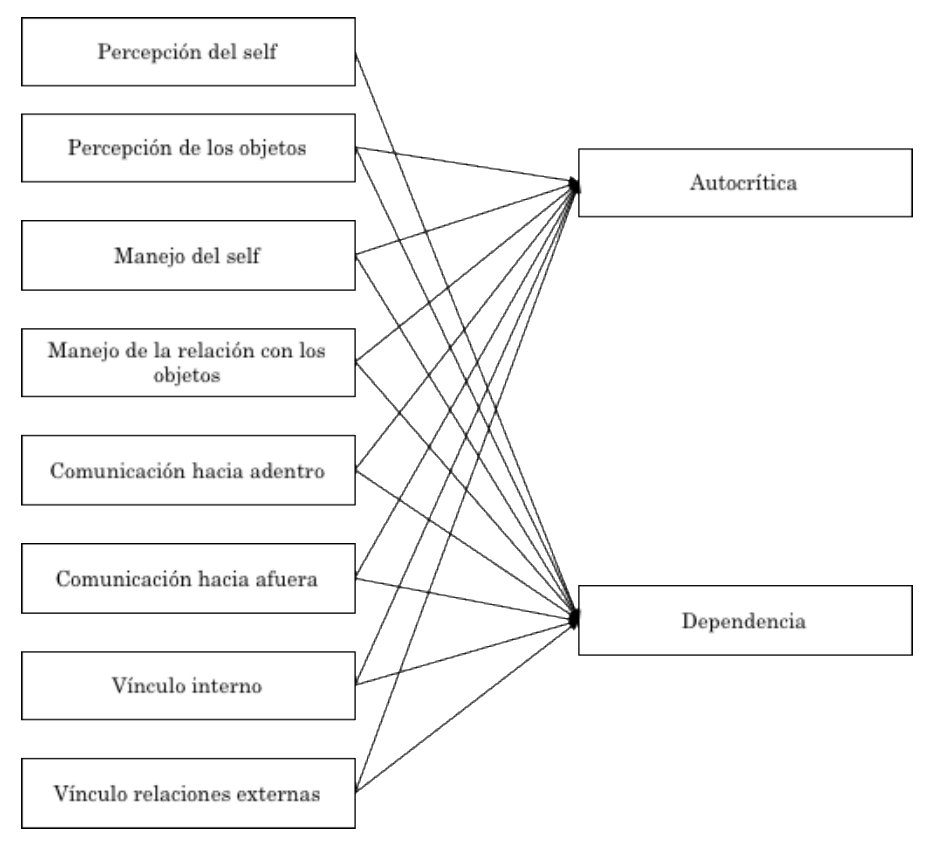

Figura 1. Diagrama resumen de las relaciones evaluadas mediante análisis de senderos. 
Finalmente, con el objetivo de estudiar el cambio en la sintomatología de los pacientes, se realizó un análisis de crecimiento latente, considerando tres mediciones del OQ, al inicio de la terapia, a la tercera sesión y a la sexta sesión, y utilizando maximum likelihood robusto como método de estimación. Dado el número de mediciones, solo se probó un modelo de crecimiento lineal. También se probó el modelo ingresando como predictores tanto del intercepto como de la pendiente las escalas de dependencia y autocrítica del DEQ. Se utilizaron los mismos criterios nombrados en el análisis de senderos para medir la bondad de ajuste del modelo. Estas variables fueron centradas previamente. Este análisis fue realizado en el programa estadístico Mplus 7 (Muthén y Muthén, 2012).

\section{Resultados}

\section{Correlaciones}

Se observó que el puntaje total en la escala de OPD-SQ presentó una correlación media con las escalas de Dependencia y Autocrítica del DEQ. Estas asociaciones son directas, indicando que mayores niveles de dependencia y autocrítica se correlacionan con una menor integración de la estructura de la personalidad. También presentó una correlación alta con los puntajes de OQ total, indicando que una peor integración de la estructura está relacionada con peor bienestar general (ver Tabla 4). Las distintas funciones del OPD-SQ presentaron un patrón similar, presentando correlaciones entre bajas y medias con Dependencia y Autocrítica y alta con el puntaje total del OQ.

Tabla 4

Correlaciones Bivariadas del Puntaje Total del OPD-SQ y Sus Subescalas con los

Resultados Terapéuticos (OQ) y el Nivel de Dependencia y Autocrítica (DEQ) Iniciales

\begin{tabular}{lccc}
\hline \multicolumn{1}{c}{ OPD-SQ y subescala } & Dependencia & Autocrítica & OQ total \\
\hline Percepción del self & $0,43^{* * * *}$ & $0,38^{* * *}$ & $0,68^{* * *}$ \\
Percepción de los objetos & $0,35^{* *}$ & $0,46^{* * *}$ & $0,62^{* * *}$ \\
Manejo del self & $0,43^{* * *}$ & $0,37^{* * *}$ & $0,74^{* * *}$ \\
Manejo de la relación con los objetos & $0,39^{* * *}$ & $0,43^{* * *}$ & $0,67^{* * *}$ \\
Comunicación emocional hacia adentro & $0,30^{* * *}$ & $0,36^{* * *}$ & $0,64^{* * *}$ \\
Comunicación emocional hacia afuera & $0,28^{* *}$ & $0,39^{* * *}$ & $0,67^{* * *}$ \\
Vínculo interno & $0,28^{* *}$ & $0,47^{* * *}$ & $0,71^{* * *}$ \\
Vínculo relaciones externas & $0,25^{*}$ & $0,29^{* *}$ & $0,53^{* * *}$ \\
OPD-SQ total & $0,39^{* * *}$ & $0,45^{* * *}$ & $0,75^{* * *}$ \\
\hline
\end{tabular}

\section{Relación entre el Estilo Depresivo y las Vulnerabilidades en el Funcionamiento Estructural}

Para evaluar la relación que existe entre las distintas vulnerabilidades en el funcionamiento estructural y los diferentes estilos depresivos, se realizó un análisis de senderos, utilizando como predictores tanto de Autocrítica como de Dependencia a todas las escalas del OPD-SQ. El modelo presentó un buen ajuste, $\chi^{2}(1, N=143)=0,12, p=0,73, R M S E A=0,00, C F I=1,00, S R M R=0,003$. En la Figura 2 se observan los senderos que resultaron significativos. Se puede ver que las vulnerabilidades en el funcionamiento estructural asociadas a la dependencia corresponden a la percepción del self y al manejo de la relación con los objetos. La autocrítica, en cambio, se relaciona con el vínculo interno.

\section{Crecimiento Latente del Bienestar General y su Relación con la Experiencia Depresiva}

Con el objetivo de estudiar el cambio en el bienestar de los pacientes, se realizó un análisis de crecimiento latente. El modelo que incluía solo el componente lineal del crecimiento presentó un buen ajuste (ver Tabla 5). Tanto el intercepto como la pendiente dieron resultados significativos, indicando que el puntaje promedio inicial de OQ total se encuentra en 75,65 puntos y que cada tres sesiones este puntaje disminuye en 5,81 puntos. 


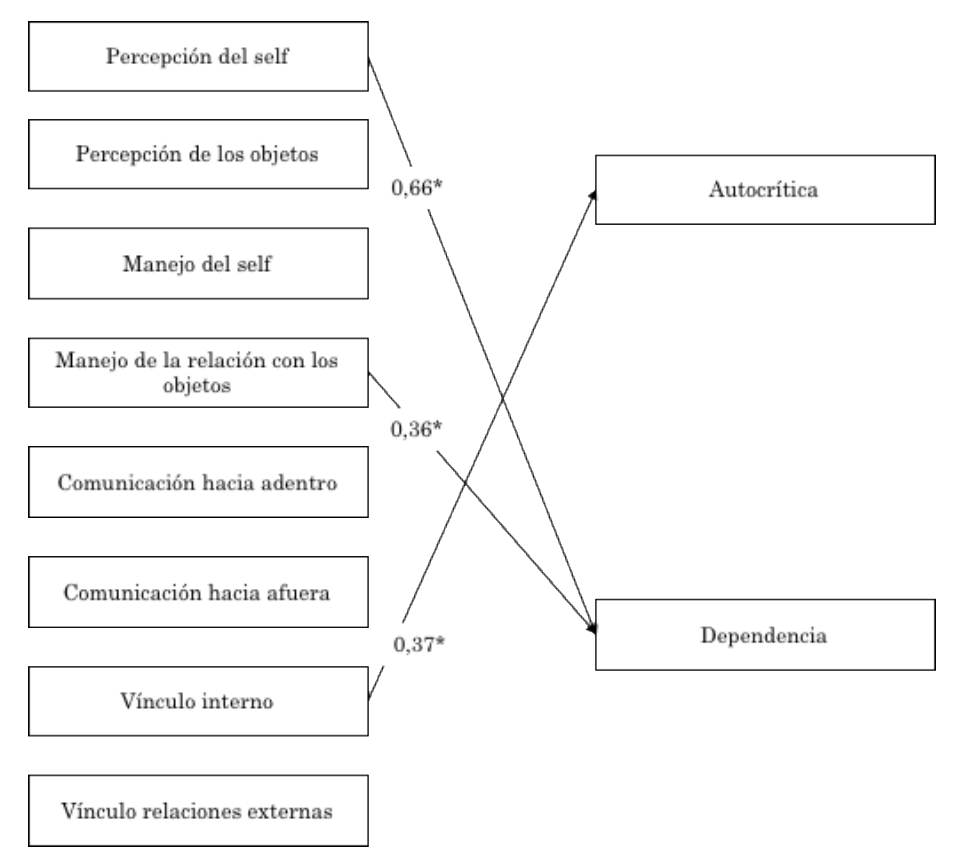

Figura 2. Relación entre vulnerabilidades del funcionamiento estructural y estilos depresivos. ${ }^{*} p<0,05$.

$\mathrm{Al}$ ingresar la dependencia y la autocrítica como predictores tanto del intercepto como de la pendiente, se observa que ambos se asocian con el intercepto, pero solo la dependencia se asocia con la pendiente. Dicho de otro modo, tanto la dependencia como la autocrítica se asocian directamente con el bienestar general inicial, lo que implica que mayores puntajes en estas escalas están relacionados con un menor bienestar general. Sin embargo, solo la dependencia se asocia con los resultados terapéuticos en el transcurso del proceso. Esta asociación es inversa, de manera que una mayor dependencia se asocia a una mayor tasa de cambio.

\section{Discusión y Conclusiones}

Este estudio examinó la relación entre las dimensiones de experiencia depresiva, vulnerabilidades en el funcionamiento estructural y resultados terapéuticos en una muestra de pacientes que presentan síntomas depresivos.

Blatt (2008) plantea que la mayor presencia de autocrítica o dependencia implica la existencia de una alteración en la sinergia dialéctica entre la autodefinición y las relaciones con los otros que acompañan desde la niñez. Refiere que la tendencia hacia un polo u otro implicaría dificultades en la gratificación durante el proceso de desarrollo y, por ende, aluden a vulnerabilidades que favorecen el desarrollo de patologías (Blatt, 2008). En este estudio, se observa que tanto la autocrítica como la dependencia se asocian con niveles menores de integración del funcionamiento estructural en términos globales y en cuanto a las funciones específicas, además de asociarse a menor bienestar subjetivo. Esto entrega evidencia a favor de lo planteado por múltiples autores: que las expresiones maladaptativas de autodefinición y relación (autocrítica y dependencia) pueden ser conceptualizadas como factores de vulnerabilidad no solo en la depresión, sino que transdiagnóstico (e.g., Blatt, 2008; Luyten \& Blatt, 2013). 
Tabla 5

Resumen de Modelos de Crecimiento Latente de los Resultados Psicoterapéuticos

\begin{tabular}{lcc}
\hline & Modelo 1 & Modelo 2 \\
\hline Intercepto & & \\
Media (Intercepto) & $75,65^{* * *}$ & $75,00^{* * *}$ \\
$\quad$ Dependencia & - & $6,21^{* *}$ \\
$\quad$ Autocrítica & - & $7,20^{* *}$ \\
Pendiente & $-5,81^{* * *}$ & $-5,85^{* * *}$ \\
Media (Intercepto) & - & $-2,62^{*}$ \\
$\quad$ Dependencia & - & 0,83 \\
$\quad$ Autocrítica & $-20,56$ & - \\
Covarianza & & \\
Intercepto-Pendiente & $1,12(1, N=143)$ & $1,93(5, N=101)$ \\
Ajuste del modelo & 0,03 & 0,00 \\
$\quad \chi^{2}$ & 1,00 & 1,00 \\
RMSEA & 0,02 & 0,01 \\
CFI & & \\
SRMR &
\end{tabular}

Nota. Modelo 1: Cambio en OQ sin predictores; Modelo 2: Cambio en OQ con subescalas de DEQ como predictores.

${ }^{*} p<0,05 ;{ }^{* *} p<0,01 ;{ }^{* * *} p<0,001$.

Específicamente, los resultados apoyan la idea de que hay vulnerabilidades en funciones estructurales específicas a la base de cada uno de los estilos de depresión, las cuales son consistentes con el funcionamiento que se espera en estos pacientes. Es así como en esta muestra se observa que altos niveles de autocrítica de los pacientes se asocian a mayores vulnerabilidades en la función de vínculos internos. Esto coincide con el supuesto de que estos pacientes parecieran no haber desarrollado objetos internos apoyadores y cuidadores, sino que más bien poseen introyectos negativos y amenazantes que transforman la realidad de manera persecutoria y exigente. En relación a la dependencia, se observa que las funciones más vulnerables se dan principalmente en relación a la percepción y manejo del self. Esto podría explicarse a partir de que los pacientes que presentan mayor dependencia pueden estar mucho más interesados en sus relaciones interpersonales que en ellos mismos. Específicamente, esta función incluye la capacidad de autorreflexión, diferenciación afectiva e identidad, todos aspectos que se esperaría que estuvieran menos integrados en estos pacientes por su dificultad de mirarse ellos mismos. Por otro lado, una segunda vulnerabilidad en el funcionamiento estructural que se encuentra relacionada a este estilo es el manejo de la relación con los objetos. Esto podría explicarse por una posible menor capacidad de contener los propios impulsos al momento de relacionarse con los otros, lo que en la experiencia depresiva se manifestaría como dependencia.

La presencia de estas vulnerabilidades específicas para los estilos depresivos lleva a pensar en la necesidad de adecuar el tratamiento, estableciendo intervenciones particulares para cada uno, como, por ejemplo, las vulnerabilidades que presentan los pacientes autocríticos en términos de su baja integración en los vínculos internos pueden hacer referencia a una pobreza de objetos internalizados capaces de cuidar, proteger y calmar. Por el contrario, es probable que estos pacientes presenten objetos amenazantes y persecutorios que a través del proceso terapéutico deben ser "silenciados". Por otro lado, las vulnerabilidades de los pacientes dependientes que girarían en torno a la percepción del self podrían ser tratadas con técnicas mentalizadoras (Luyten, Fonagy, Lemma \& Target, 2012). La disminución de la capacidad en el manejo de la relación con los objetos podría eventualmente trabajarse en conexión con la función de percepción del self. Para ello, es importante que el terapeuta sea capaz de ayudar al paciente a regular su relación con los demás (objetos) y esto se puede lograr favoreciendo en el paciente un yo observador, es decir, un yo que sea capaz de mirarse con cierta distancia (Rudolf, 2013), para poder observar de manera más consciente la forma impulsiva de actuar frente al resto y, con ello, ser capaz de resolver las tormentas emocionales, reconociendo y validando los propios intereses. 
Otro de los resultados es la relevancia que tiene la dependencia y la autocrítica en el bienestar general de los pacientes al inicio de sus procesos terapéuticos. Se observa que los pacientes que presentan un alto nivel de dependencia o de autocrítica comienzan sus terapias con un nivel menor de bienestar subjetivo global, el cual incluye sintomatología ansiosa y depresiva y malestar en las relaciones interpersonales y en el rol social. Esto coincide con la evidencia empírica y clínica anterior que muestra cómo una alta presencia de autocrítica o dependencia es producto de disrupciones en el desarrollo que han llevado a un desbalance y, si el desbalance es hacia la dependencia, aparecen sentimientos de incertidumbre con los demás, alienación, soledad y aislamiento; en cambio, si el desbalance es hacia el autovalor aparecen temores básicos de aniquilación, preocupaciones acerca de la pérdida de control, sentimientos de vergüenza, culpa e inferioridad. Todo esto implica menor bienestar subjetivo, el cual pareciera confirmarse con los resultados obtenidos (Blatt, 1990, 1995; Blatt \& Blass, 1990, 1996; Blatt \& Shichman, 1983).

Además de que las experiencias depresivas muestran su influencia en el bienestar inicial, se observa que, consistente con otros estudios (Blatt, 1992; Blatt et al., 1988; Blatt \& Felsen, 1993; Blatt \& Ford, 1994; Sotsky et al., 1991), los resultados apoyan la importancia de estas en la evolución del tratamiento. En el caso del presente estudio, se observó que el estilo depresivo dependiente tiene un efecto sobre la evolución, específicamente, que a mayor presencia de dependencia mayor es la tasa de cambio en los resultados terapéuticos. Esto no sucede con la presencia de autocrítica, la cual no presenta un efecto significativo en la evolución de estos. Este resultado desconfirma la hipótesis inicial; sin embargo, al observar las trayectorias y aunque estas no resultaron ser significativas para los dependientes, sí se observa que no avanzan como lo hacen los dependientes, por lo que vale la pena seguir investigando sobre aquello. El cambio en los resultados terapéuticos de los pacientes con estilo dependiente puede entenderse, ya que los pacientes dependientes están ávidos de vincularse, lo que provoca una mayor permeabilidad a las intervenciones. Esto podría explicar su capacidad de beneficiarse de diferentes terapias e intervenciones (Eames \& Roth, 2000; Kanninen, Salo \& Punamäki, 2000). De hecho, son pacientes que sufren de inseguridades en el apego y necesitan fuertemente de apoyo interpersonal para evitar los agudos sentimientos de fragilidad y soledad (Mongrain \& Leather, 2006).

Este estudio viene a apoyar la importancia de las características particulares de los pacientes, tanto al inicio de los procesos como su influencia en la evolución de estos. Esto evidencia un cambio en la forma de investigar en psicoterapia, ya no comparando diversos tratamientos que resultan útiles para la reducción de un/os síntoma/s en particular (e.g., depresión), sino que abarcando temas más complejos, como identificando las características particulares de los pacientes, diferenciando el tipo de tratamiento más efectivo, bajo qué condiciones y con qué tipo de pacientes (Blatt et al., 2002; Blatt \& Shahar, 2004; Paul, 1969).

Entre las limitaciones de este estudio, es necesario considerar que no se contó con un grupo control ni se consideró para los análisis la presencia de otros diagnósticos en comorbilidad; hacerlo permitiría identificar con mayor claridad si las limitaciones estructurales específicas tienen que ver con la depresión o con la patología en general. También, al no existir datos longitudinales sobre la génesis de la sintomatología, solo se pudo evaluar la asociación entre las vulnerabilidades y las experiencias depresivas, quedando fuera la posibilidad de establecer asociaciones causales entre las mismas. Un estudio descriptivo, en el que se estudien las vulnerabilidades estructurales en conjunto con la sintomatología y las distintas experiencias depresivas, podría dar más luz sobre la asociación temporal entre estas variables. Por otro lado, fueron consideradas solo tres mediciones del bienestar subjetivo. Resultaría relevante considerar mayor cantidad de mediciones (ojalá sesión a sesión) para realizar un análisis más detallado de la evolución, ya que algunos autores plantean que ambos tipos de experiencia depresiva cambian de manera diferente y en distintos momentos del proceso terapéutico (Eames \& Roth, 2000; Fertuck, Bucci, Blatt \& Ford, 2004; Shapiro et al., 1999). Por último, resultaría interesante poder contar con psicoterapias de larga duración, ya que así se esperaría encontrar una evolución positiva de cambio en pacientes con alta presencia de autocrítica.

\section{Referencias}

Alvarado, R., Vega, J., Sanhueza, G. \& Muñoz, M. G. (2005). Evaluación del Programa para la Detección, Diagnóstico y Tratamiento Integral de la Depresión en atención primaria, en Chile. Revista Panamericana de Salud Pública, 18, 278-286. https://doi.org/10.1590/S1020-49892005000900008

Andrade, L., Caraveo-Anduaga, J. J., Berglund, P., Bijl, R. V., De Graaf, R., Vollebergh, W. ... Wittchen, H. -U. (2003). The epidemiology of major depressive episodes: Results from the International Consortium of Psychiatric Epidemiology (ICPE) surveys. International Journal of Methods in Psychiatric Research, 12, 3-21. https://doi.org/10.1002/mpr.138

Beck, A. T., Steer, R. A. \& Carbin, M. G. (1988). Psychometric properties of the Beck Depression Inventory: Twenty-five years of evaluation. Clinical Psychology Review, 8, 77-100. https://doi.org/10.1016/0272-7358(88)90050-5 
Beck, A. T., Ward, C. H., Mendelson, M., Mock, J. \& Erbaugh, J. (1961). An inventory for measuring depression. Archives of General Psychiatry, 4, 561-571. https://doi.org/10.1001/archpsyc.1961.01710120031004

Blatt, S. J. (1974). Levels of object representation in anaclitic and introjective depression. The Psychoanalytic Study of the Child, 29, 107-157. https://doi.org/10.1080/00797308.1974.11822616

Blatt, S. J. (1990). Interpersonal relatedness and self-definition: Two personality configurations and their implications for psychopathology and psychotherapy. En J. L. Singer (Ed.), Repression and dissociation: Implications for personality theory, psychopathology and health (pp. 299-335). Chicago, IL: University of Chicago Press.

Blatt, S. J. (1991). A cognitive morphology of psychopathology. The Journal of Nervous and Mental Disease, 179, 449-458. https://doi.org/10.1097/00005053-199108000-00001

Blatt, S. (1992). The differential effect of psychotherapy and psychoanalysis with anaclitic and introjective patients: The Menninger Psychotherapy Research Project revisited. Journal of the American Psychoanalytic Association, 40, 691-724. https://doi.org/10.1177/000306519204000303

Blatt, S. J. (1995). Representational structures in psychopathology. En D. Cicchetti \& S. L. Toth (Eds.), Rochester symposium on developmental psychopathology: Vol. 6. Emotion, cognition, and representation (pp. 1-33). Rochester, NY: University of Rochester Press.

Blatt, S. J. (2007). A fundamental polarity in psychoanalysis: Implications for personality development, psychopathology, and the therapeutic process. Psychoanalytic Inquiry, 26, 494-520. https://doi.org/10.1080/07351690701310581

Blatt, S. J. (2008). Polarities of experience: Relatedness and self-definition in personality development, psychopathology, and the therapeutic process. Washington, DC: American Psychological Association. https://doi.org/10.1037/11749-000

Blatt, S. J. \& Blass, R. B. (1990). Attachment and separateness: A dialectic model of the products and processes of development throughout the life cycle. The Psychoanalytic Study of the Child, 45, 107-127. https://doi.org/10.1080/00797308.1990.11823513

Blatt, S. J. \& Blass, R. B. (1996). Relatedness and self definition: A dialectic model of personality development. En G. G. Noam \& K. W. Fischer (Eds.), Development and vulnerability in close relationships (pp. 309-338). Hillsdale, NJ: Lawrence Erlbaum.

Blatt, S. J., D'Afflitti, J. P. \& Quinlan, D. M. (1976). Experiences of depression in normal young adults. Journal of Abnormal Psychology, 85, 383-389. https://doi.org/10.1037/0021-843X.85.4.383

Blatt, S. \& Felsen, I. (1993). Different kinds of folks may need different kinds of strokes: The effect of patients' characteristics on therapeutic process and outcome. Psychotherapy Research, 3, 245-259. https://doi.org/10.1080/10503309312331333829

Blatt, S. J., Ford, R. Q., Berman Jr., W. H., Cook, B., Cramer, P. \& Robins, C. E. (1994). Therapeutic change: An object relations perspective. https://doi.org/10.1007/978-1-4899-1010-3

Blatt, S. J., Ford, R. Q., Berman, W., Cook, B. \& Meyer, R. (1988). The assessment of change during the intensive treatment of borderline and schizophrenic young adults. Psychoanalytic Psychology, 5, 127-158. https://doi.org/10.1037/0736-9735.5.2.127

Blatt, S. J., Quinlan, D. M., Pilkonis, P. A. \& Shea, M. T. (1995). Impact of perfectionism and need for approval on the brief treatment of depression: The National Institute of Mental Health Treatment of Depression Collaborative Research Program revisited. Journal of Consulting and Clinical Psychology, 63, 125-132. https://doi.org/10.1037/0022-006X.63.1.125

Blatt, S. J. \& Shahar, G. (2004). Das dialogische Selbst: Adaptive und maladaptive Dimensionen [El self dialógico: dimensiones adaptivas y maladaptivas]. En P. Giampieri-Deutsch (Ed.), Psychoanalyse im Dialog der Wissenschaften. Band 2: Anglo-Amerikanische Perspektiven [Psicoanálisis en el diálogo de las ciencias. Volumen 2: Perspectivas anglo-americanas] (pp. 285-309). Stuttgart, Alemania: Kohlhammer.

Blatt, S. J., Shahar, G. \& Zuroff, D. C. (2002). Anaclitic/sociotropic and introjective/autonomous dimensions. En J. C. Norcross (Ed.), Psychotherapy relationships that work: Therapist contributions and responsiveness to patients (pp. 315-334). New York, NY: Oxford University Press.

Blatt, S. J. \& Shichman, S. (1983). Two primary configurations of psychopathology. Psychoanalysis and Contemporary Thought, 6, 187-254. Extraído de http://psycnet.apa.org/record/1984-15126-001

Blatt, S. J., Zuroff, D. C., Bondi, C. M., Sanislow III, C. A. \& Pilkonis, P. A. (1998). When and how perfectionism impedes the brief treatment of depression: Further analyses of the National Institute of Mental Health Treatment of Depression Collaborative Research Program. Journal of Consulting and Clinical Psychology, 66, 423-428. https://doi.org/10.1037//0022-006X.66.2.423

Blatt, S. J., Zuroff, D. C., Quinlan, D. M. \& Pilkonis, P. A. (1996). Interpersonal factors in brief treatment of depression: Further analysis of the National Institute of Mental Health Treatment of Depression Collaborative Research Program. Journal of Consulting and Clinical Psychology, 64, 162-171. https://doi.org/10.1037/0022-006X.64.1.162

Chile, Ministerio de Salud (2013). Guía clínica AUGE: depresión en personas de 15 años y más (2a ed.). Santiago, Chile: Autor, Subsecretaría de Salud Pública. Extraído de http://www.minsal.cl/portal/url/item/7222754637c08646e04001011f014e64.pdf

Corveleyn, J., Luyten, P. \& Blatt, S. J. (Eds.) (2005). The theory and treatment of depression: Towards a dynamic interactionism model. Leuven, Bélgica: Leuven University Press.

Dagnino, P., Gómez-Barris, E., Gallardo, A. M., Valdés, C. \& de la Parra, G. (2017). Dimensiones de la experiencia depresiva y funcionamiento estructural: ¿qué hay en la base de la heterogeneidad de la depresión? Revista Argentina de Clínica Psicológica, 26, 83-94. https://doi.org/10.24205/03276716.2017.1007

Dagnino, P., Pérez, C., Gómez, A., Gloger, S. \& Krause, M. (2017). Depression and attachment: How do personality styles and social support influence this relation? Research in Psychotherapy: Psychopathology, Process and Outcome, 20, 53-62. https://doi.org/10.4081/ripppo.2017.237

Dahlbender, R. W., Rudolf, G. \& OPD Task Force (2006). Psychic structure and mental functioning: Current research on the reliable measurement and clinical validity of operationalized psychodynamic diagnostics (OPD) system. En PDM Task Force, Psychodynamic diagnostic manual (pp. 615-662). Silver Spring, MD: Alliance of Psychoanalytic Organizations.

de la Parra, G., Dagnino, P., Valdés, C. \& Krause, M. (2017). Beyond self-criticism and dependency: Structural functioning of depressive patients and its treatment. Research in Psychotherapy: Psychopathology, Process and Outcome, 20, 43-52. https://doi.org/10.4081/ripppo.2017.236

de la Parra, G., Undurraga, C., Crempien, C., Valdés, C., Dagnino, P. \& Gómez-Barris, E. (2018). Estructura de personalidad en pacientes con depresión: adaptación de un instrumento y resultados preliminares. Psykhe, 27(2), 1-20. https://doi.org/10.7764/psykhe.27.2.1133

de la Parra, G. \& von Bergen, A. (2001, Junio). Administration of the Outcome Questionnaire OQ-45.2 in Santiago de Chile: Validity, reliability, applicability, normative data and clinical projections. Ponencia presentada en la Society for Psychotherapy Research (SPR) 32 $2^{\text {nd }}$ International Annual Meeting, Montevideo, Uruguay. 
de la Parra, G., von Bergen, A. \& del Río, M. (2002). Primeros hallazgos de la aplicación de un instrumento que mide resultados psicoterapéuticos en una muestra de pacientes y de población general. Revista Chilena de Neuro-Psiquiatría, 40, 201-209. https://doi.org/10.4067/S0717-92272002000300003

Dinger, U., Schauenburg, H., Hörz, S., Rentrop, M., Komo-Lang, M., Klinkerfuß, M. ... Ehrenthal, J. C. (2014). Self-report and observer ratings of personality functioning: A study of the OPD system. Journal of Personality Assessment, 96, 220-225. https://doi.org/10.1080/00223891.2013.828065

Doering, S., Burgmer, M., Heuft, G., Menke, D., Bäumer, B., Lübking, M. ... Schneider, G. (2014). Assessment of personality functioning: Validity of the operationalized psychodynamic diagnosis axis IV (structure). Psychopathology, 47, 185-193. https://doi.org/10.1159/000355062

Eames, V. \& Roth, A. (2000). Patient attachment orientation and the early working alliance: A study of patient and therapist reports of alliance quality and ruptures. Psychotherapy Research, 10, 421-434. https://doi.org/10.1093/ptr/10.4.421.

Ehrenthal, J. C., Dinger, U., Horsch, L., Komo-Lang, M., Klinkerfuß, M., Grande, T. \& Schauenburg, H. (2012). Der OPDStrukturfragebogen (OPD-SF): Erste Ergebnisse zu Reliabilität und Validität [El Cuestionario Estructural OPD (OPD-SQ): Primeros resultados de confiabilidad y validez]. Psychotherapie, Psychosomatik, Medizinische Psychologie, 62, 25-32. https://doi.org/10.1055/s-0031-1295481

Fertuck, E. A., Bucci, W., Blatt, S. J. \& Ford, R. Q. (2004). Verbal representation and therapeutic change in anaclitic and introjective inpatients. Psychotherapy: Theory, Research, Practice and Training, 41, 13-25. https://doi.org/10.1037/0033-3204.41.1.13

Fischer, K. W., Ayoub, C., Singh, I., Noam, G., Maraganore, A. \& Raya, P. (1997). Psychopathology as adaptive development along distinctive pathways. Development and Psychopathology, 9, 749-779. https://doi.org/10.1017/s0954579497001429

Grupo de Trabajo OPD (2008). Diagnóstico psicodinámico operacionalizado (OPD-2). Manual para el diagnóstico, indicación y planificación de la psicoterapia. Barcelona, España: Herder.

Jackson, D. L., Gillaspy Jr., J. A. \& Purc-Stephenson, R. (2009). Reporting practices in confirmatory factor analysis: An overview and some recommendations. Psychological Methods, 14, 6-23. https://doi.org/10.1037/a0014694

Kanninen, K., Salo, J. \& Punamäki, R. -L. (2000). Attachment patterns and working alliance in trauma therapy for victims of political violence. Psychotherapy Research, 10, 435-449. https://doi.org/10.1093/ptr/10.4.435

Lambert, M. J., Hansen, N. B., Umphress, V., Lunnen, K., Okiishi, J., Burlingame, G. M. ... Reisinger, C. W. (1996). Administration and scoring manual for the Outcome Questionnaire (OQ-45.2). Wilmington, DE: American Professional Credentialing Services.

Luyten, P. \& Blatt, S. J. (2011). Integrating theory-driven and empirically-derived models of personality development and psychopathology: A proposal for DSM V. Clinical Psychology Review, 31, 52-68. https://doi.org/10.1016/j.cpr.2010.09.003

Luyten, P. \& Blatt, S. J. (2013). Interpersonal relatedness and self-definition in normal and disrupted personality development: Retrospect and prospect. American Psychologist, 68, 172-183. https://doi.org/10.1037/a0032243

Luyten, P., Fonagy, P., Lemma, A. \& Target, M. (2012). Depression. En A. Bateman \& P. Fonagy (Eds.), Handbook of mentalizing in mental health practice (pp. 385-417). Washington, DC: American Psychiatric Association.

Mongrain, M. \& Leather, F. (2006). Immature dependence and self- criticism predict the recurrence of major depression. Journal of Clinical Psychology, 62, 705-713. https://doi.org/10.1002/jclp.20263

Muthén, L. K. \& Muthén, B. O. (2012). Mplus: Statistical analysis with latent variables (Versión 7) [Software computacional]. Los Angeles, CA: Autores.

Paul, G. L. (1969). Behavior modification research: Design and tactics. En C. M. Franks (Ed.), Behavior therapy: Appraisal and status (pp. 29-62). New York, NY: McGraw Hill.

Pérez, J. C., Morales, S., Dagnino, P., Silva, J., Rodríguez, E., Echávarri, O. ... Krause, M. (2018). Depreciating sense of self, pathological dependency and concernedness: A new factor solution of the Depressive Experience Questionnaire (DEQ). Manuscrito en preparación.

$\mathrm{R}$ Development Core Team (2008). $R$ : A language and environment for statistical computing. Wien, Austria: R Foundation for Statistical Computing.

Rudolf, G. (2013). Strukturbezogene Psychotherapie: Leitfaden zur psychodynamischen Therapie struktureller Störungen [Psicoterapia estructural: una guía para la terapia psicodinámica de los trastornos estructurales] ( $3^{a}$ ed.). Stuttgart, Alemania: Schattauer Verlag.

Rudolf, G., Grande, T., Oberbracht, C. \& Jakobsen, T. (1996). Erste empirische Untersuchungen zu einem neuen diagnostischen System: Die Operationalisierte Psychodynamische Diagnostik (OPD) [Primeras investigaciones empíricas acerca de un nuevo sistema diagnóstico: el Diagnóstico psicodinámico operacionalizado (OPD)]. Zeitschrift für Psychosomatische Medizin und Psychoanalyse, 42, 343-357. Extraído de https://www.jstor.org/stable/23997350

Rudolf, G., Oberbracht, C. \& Grande, T. (1998). Die Struktur-Checkliste. Ein anwenderfreundliches Hilfsmittel für die Strukturdiagnostik nach OPD [La lista de estructura: una herramienta fácil para el diagnóstico estructural según OPD]. En H. Schauenburg, H. J. Freyberger, M. Cierpka \& P. Buchheim (Eds.), OPD in der Praxis. Konzepte, Anwendungen, Ergebnisse der operationalisierten psychodynamischen Diagnostik [OPD en la práctica. Conceptos, aplicación, resultados del diagnóstico psicodinámico operacionalizado] (pp. 167-181). Bern, Suiza: Huber.

Shapiro, D., Hardy, G., Aldridge, J., Davidson, C., Rowe, C. \& Reilly, S. (1999). Therapist responsiveness to client attachment styles and issues observed in client-identified significant events in psychodynamic-interpersonal psychotherapy. Psychotherapy Research, 9 , 36-53. https://doi.org/10.1080/10503309912331332581

Sotsky, S. M., Glass, R. B. J., Shea, M. T., Pilkonis, P. A., Collins, J. F., Elkin, I. ... Moyer, J. (1991). Patient predictors of response to psychotherapy and pharmacotherapy: Findings in the NIMH Treatment of Depression Collaborative Research Program. The American Journal of Psychiatry, 148, 997-1008. https://doi.org/10.1176/ajp.148.8.997

Sroufe, L. A. (1997). Psychopathology as an outcome of development. Development and Psychopathology, 9, 251-268. https://doi.org/10.1017/S0954579497002046

Valdés, C. \& Errázuriz, P. (2012). Salud mental en Chile: el pariente pobre del sistema de salud (Claves de Políticas Públicas No 11). Santiago, Chile: Universidad Diego Portales, Instituto de Políticas Públicas. Extraído de https://fundacionsaludmental.cl/wpcontent/uploads/2018/03/Salud_Mental_en_Chile.pdf

Valdés, C., Morales-Reyes, I., Pérez, J. C., Medellín, A., Rojas, G. \& Krause, M. (2017). Propiedades psicométricas del Inventario de Depresión de Beck IA para la población chilena. Revista Médica de Chile, 145, 1005-1012. https://doi.org/10.4067/s003498872017000801005

van Praag, H. M. (2010). No functional psychopharmacology without functional psychopathology. Acta Psychiatrica Scandinavica, 122, 438-439. https://doi.org/10.1111/j.1600-0447.2010.01629.x 
Wallerstein, R. S. (1986). Forty-two lives in treatment: A study of psychoanalysis and psychotherapy. New York, NY: Guilford Press.

Zuroff, D. C., Blatt, S. J., Sotsky, S. M., Krupnick, J. L., Martin, D. J., Sanislow III, C. A. \& Simmens, S. (2000). Relation of therapeutic alliance and perfectionism to outcome in brief outpatient treatment of depression. Journal of Consulting and Clinical Psychology, 68, 114-124. https://doi.org/10.1037/0022-006X.68.1.114

Zuroff, D. C., Quinlan, D. M. \& Blatt, S. J. (1990). Psychometric properties of the Depressive Experiences Questionnaire in a college population. Journal of Personality Assessment, 55, 65-72. https://doi.org/10.1080/00223891.1990.9674047

Fecha de recepción: Diciembre de 2016.

Fecha de aceptación: Mayo de 2018. 